\title{
Spacetime quantization illustrates general relativity and quantum theory
}

\author{
Yazeed Turki Alharbi
}

KFUPM, KSA

Abstract:

Many experiments and collected data support general relativity as it explains spacetime as a smooth Riemannian manifold. However, general relativity does not explain the chaotic nature of spacetime on the quantum level, where determinism is almost impossible. This is one of the major problems in physics yet it requires a deeper theory to solve this incompatibility. In this research, a derivation made from Newton's law of gravitation to better explain the behavior of spacetime in the quantum world with the assumption that spacetime is quantized with energy waveparticles and these wave-particles accumulate to form massive wave like particles (hence the standard model of particle physics). In the end, the derivation and the assumption together clearly illustrate general relativity and quantum theory.

Derivation and assumption:

The assumption is that spacetime is quantized with wave like particles and these wave like particles accumulate to form massive wave like particles. A single space time quantum would have an energy of:

$$
E=h v=h\left(1\left(\frac{1}{s}\right)\right)=h_{0}
$$

Also, a single spacetime quantum would move a distance $\Delta x$ in the accumulation process that can be derived from newton's gravitational law:

$$
\Delta x=\iint_{t}^{\infty} \frac{G m}{r^{2}} d t d t
$$

The wave like spacetime quanta are assumed to travel at the speed of light, therefore: 


$$
\begin{gathered}
\Delta x=\iint_{t}^{\infty} \frac{G m}{(c t)^{2}} d t d t \\
\Delta x=\frac{G m}{c^{2}}\left|\ln \frac{r}{c}\right|
\end{gathered}
$$

Another assumption to be made intuitively regarding the accumulation process is that space time wave like particles are connected. The movement of a single particle causes the movement of another. That means a volume of an $\mathrm{N}$ number of particles accumulate $\mathrm{N}$ number of particles in more volume. The accumulation of the particles can be expressed as the pure energy of a massive particle:

$$
m c^{2}=n h_{0}+n \frac{h_{0}}{L} \frac{G m}{c^{2}}\left|\ln \frac{r}{c}\right|
$$

Where $\frac{h_{0}}{L}$ is the linear energy density of vacuum. However, the energy density of the massive particle remains constant and equals to the energy density of the vacuum and whether its value is static or not is not a relevant argument in this context. Therefore:

$$
\begin{gathered}
\frac{n h_{0}+n \frac{h_{0}}{L} \frac{G m}{c^{2}}\left|\ln \frac{r}{c}\right|}{\frac{4}{3} \pi r^{3}}=3\left|\frac{h_{0}}{L}\right| \\
n L+n \frac{G m}{c^{2}}\left|\ln \frac{r}{c}\right|=4 \pi r^{3} \\
e^{n L} *\left(\frac{r}{c}\right)^{\frac{n G m}{c^{2}}}=e^{4 \pi r^{3}} \\
e^{n L}=\left(\frac{c}{r}\right)^{\frac{n G m}{c^{2}}} e^{4 \pi r^{3}} \\
e^{n L}=\left(\frac{c}{r}\right)^{\frac{n G m}{c^{2}}} \\
e^{n L} r^{\frac{n G m}{c^{2}}}=c^{\frac{n G m}{c^{2}}}
\end{gathered}
$$




$$
r=c e^{-\frac{L c^{2}}{G m}}
$$

Consequently, according to equation (7):

$$
\begin{gathered}
c e^{-\frac{L c^{2}}{G m}}=\sqrt[3]{\frac{m c^{2} L}{3 h_{0}} \frac{3}{4 \pi}} \\
\therefore L=2.576 * 10^{-56} \mathrm{~m}
\end{gathered}
$$

Also, since an expression of $r$ is known:

$$
m c^{2}=n h_{0}+n \frac{h_{0}}{L} \frac{G m}{c^{2}}\left|\ln \frac{r}{c}\right|=2 n h_{0}
$$

\section{Results:}

\section{1- Proton's mass:}

The mass of the proton can be expressed as the whole energy of the accumulated spacetime particles made by the quarks and the electron as described earlier for the accumulation processes. However, it can be calculated using the notation for the electron's wavelength in the first orbit since it resembles the energy provided to the electron from the quarks which is the is the same pure energy for the proton:

$$
\frac{h}{m v}=N * n \frac{m c^{2}}{h_{0}} L
$$

Where $\mathrm{n}$ is the effect of the many body problem on the electron from the quarks' accumulation processes. $\mathrm{N}$ is the repetition for one oscillation. Therefore, the energy of the proton can be calculated as:

$$
E_{p}=N h_{0}
$$

Also, the energy resultant from an oscillation of the accumulation processes is equal to:

$$
E=k_{u} m_{u} c^{2}+\frac{1}{2} k_{d} m_{d} c^{2}, \quad k_{u}, k_{d} \in W
$$

Which means:

$$
E_{p}=\frac{h_{0}^{2} h}{m^{2} c^{2} v L n}=N h_{0}=n E
$$


According to the assumption, by trying the possible values of $E$ we would end up with the mass of the proton. Therefore:

$$
\begin{gathered}
\frac{h_{0}^{2} h}{m^{2} c^{2} v L n}=n\left(m_{u} c^{2}\right) \\
n=\sqrt[2]{\frac{h_{0}^{2} h}{m_{e}^{2} c^{2} v L\left(m_{u} c^{2}\right)}}
\end{gathered}
$$

Substituting $\mathrm{n}$ :

$$
\begin{aligned}
& E_{p}=\left|\frac{h_{0}^{2} h}{m^{2} c^{2} v L n}\right| \\
& M_{p}=\frac{h_{0} h^{\frac{1}{2}}}{m_{e} c^{2}} \sqrt[2]{\frac{m_{u}}{v_{e} L}}
\end{aligned}
$$

And since the up quark's mass is $1.7-3.3 \mathrm{MeV}$ :

$$
M_{p}=\frac{h_{0} h^{\frac{1}{2}}}{m_{e} c^{2}} \sqrt[2]{\frac{m_{u}}{v_{e} L}}=1.529-2.130 * 10^{-27} \mathrm{~kg}
$$

And here it is. The mass of the proton approximated using physical constants. 


\section{2- Elements' absorption and emission spactra:}

Finally, it is possible to examine the assumptions by its prediction of the spacetime behavior on the quantum level resembling absorption and emission spectra's wavelengths of many elements using the equation:

$$
\frac{1}{|v|} \frac{2 \pi\left(n_{k_{f}} r_{n_{f}}-n_{k_{i}} r_{n_{i}}\right) m v}{h} \frac{m c^{2}}{h_{0}} h_{0}=h f
$$

Since the accumulation process of the electron pulls $\frac{n_{k} m c^{2}}{h_{0}} \frac{2 \pi r m v}{h}$ number of spacetime particles in one oscillation, for a single oscillating spacetime particle which it's oscillation like an electron's accumulated spacetime particles' oscillation, it must be multiplied by an integer to be equal to the difference between the total amount of particles accumulated in two radii and that integer is the velocity's magnitude of the electron.

$$
\begin{gathered}
\frac{1}{Z} \frac{2 \pi}{h} a_{B} n_{k} \frac{m^{2} c^{2}}{h_{0}} h_{0}\left(n_{k_{f}} n_{f}^{2}-n_{k_{i}} n_{i}^{2}\right)=h f \\
y=\frac{2 \pi}{h} a_{B} m^{2} c^{2}=\text { constant }=3.740820925 * 10^{-20} \\
y \frac{n_{k_{f}} n_{f}^{2}-n_{k_{i}} n_{i}^{2}}{Z}=h f \\
\kappa=h c \frac{Z}{y\left(n_{k_{f}} n_{f}^{2}-n_{k_{i}} n_{i}^{2}\right)} \\
\Lambda^{\prime}=5.310130761 * 10^{-6} \frac{Z}{\left(n_{k_{f}} n_{f}^{2}-n_{k_{i}} n_{i}^{2}\right)}
\end{gathered}
$$

From another perspective, the calculations suggest that for every element, its' absorption and emission spectra's wavelengths can possibly be one of the values of this equation:

$$
\Lambda^{\prime}=5.310130761 * 10^{-6} \frac{Z}{N}, \quad N \in Z^{+}
$$




\begin{tabular}{|c|l|l|l|l|l|l|l|l|}
\hline Element & \multicolumn{5}{|c|}{ K(nm) } & \multicolumn{4}{c|}{$N$} \\
\hline $\mathrm{H}$ & 410 & 434 & 486 & 656 & 13 & 12 & 11 & 8 \\
\hline $\mathrm{Na}$ & 499 & 572 & 590 & 615 & 117 & 102 & 99 & 95 \\
\hline $\mathrm{Ca}$ & 435 & 559 & 590 & 640 & 244 & 190 & 180 & 166 \\
\hline $\mathrm{Mg}$ & 494 & 545 & 580 & 618 & 129 & 117 & 110 & 103 \\
\hline $\mathrm{Ne}$ & 483 & 542 & 640 & 697 & 110 & 120 & 83 & 76 \\
\hline
\end{tabular}

Table I: All the values for the wavelengths of the emission and absorption spectra for all the elements are constants divided by integers as predicted.

\section{Conclusion:}

The derived assumptions on the quantum nature of spacetime reveals that the chaotic fluctuations of spacetime in the presence of elementary particles are the just the spacetime wavelike particles being pulled in different directions due to the accumulation processes of massive fundamental particles. The most important outcome of these assumptions that spacetime particles are quantized and therefore predictable as in the calculations regarding the absorption and the emission spectra of elements and the approximation of the proton's mass using physical constants. In larger scales, spacetime particles are being pulled towards the center of an object which means that they are oriented into a single point in space, and that's why general relativity is considering spacetime as a smooth Riemannian manifold. 
References:

1) Britannica, Newton's law of gravity. https://www.britannica.com/science/gravity-physics/Newtonslaw-of-gravity

2) Desmos, Web app application, https://www.desmos.com/calculator

3) Honolulu community college science site 122 , The absorption and emission spectra of certain elements. http://www.chem1.com/acad/webtext/atoms/atptimages/atomic_line_spectra.png 